\title{
Líneas de calor para convección natural en cavidades cerradas: Tres casos de estudio
}

\section{Heatlines in Free Convection within Closed Cavities: Three Study Cases}

Cervantes de Gortari J.G.

Departamento de Termoenergía

División de Ingeniería Mecánica e Industrial

Facultad de Ingeniería, Universidad Nacional Autónoma de México

E-mail:jgonzalo@servidor.unam.mx

Torchia-Núñez J.C.

Departamento de Termoenergía

División de Ingeniería Mecánica e Industrial

Facultad de Ingeniería, Universidad Nacional Autónoma de México

E-mail:tadeo_isidoro_cruz@yahoo.com

Serrano-Gutiérrez J.A.

Departamento de Termoenergía

División de Ingeniería Mecánica e Industrial

Facultad de Ingeniería, Universidad Nacional Autónoma de México

E-mail:jaserranog@hotmail.com

Muñoz-Barrera A.

Departamento de Termoenergía

División de Ingeniería Mecánica e Industrial

Facultad de Ingeniería, Universidad Nacional Autónoma de México

E-mail:fijamb21@yahoo.com.mx

Guzmán-Vázquez E.

Departamento de Termoenergía

División de Ingeniería Mecánica e Industrial

Facultad de Ingeniería, Universidad Nacional Autónoma de México

E-mail:tadeo_isidoro_cruz@yahoo.com

Gutiérrez-Coria F.

Departamento de Termoenergía

División de Ingeniería Mecánica e Industrial

Facultad de Ingeniería, Universidad Nacional Autónoma de México

E-mail:fdg_coria@yahoo.com.mx

Chávez-López $\mathrm{O}$.

Departamento de Termoenergía

División de Ingeniería Mecánica e Industrial

Facultad de Ingeniería, Universidad Nacional Autónoma de México

E-mail:tadeo_isidoro_cruz@yahoo.com 
DOI: http://dx.doi.org/10.22201/fi.25940732e.2010.11n4.031

Líneas de calor para convección natural en cavidades cerradas: Tres casos de estudio

\author{
Rosas-Yescas I. \\ Departamento de Termoenergía \\ División de Ingeniería Mecánica e Industrial \\ Facultad de Ingeniería, Universidad Nacional Autónoma de México \\ E-mail:isroye@yahoo.com.mx \\ Martínez-Galván E. \\ Departamento de Termoenergía \\ División de Ingeniería Mecánica e Industrial \\ Facultad de Ingeniería, Universidad Nacional Autónoma de México \\ E-mail:holallo@hotmail.com
}

(Recibido: junio de 2006; reevaluado: febrero y agosto de 2009; aceptado: mayo de 2010)

\title{
Resumen
}

Se presenta la técnica de visualización de líneas de calor para tres casos específicos de convección natural en cavidades cerradas. El objetivo es mostrar las ventajas de visualizar la transferencia de calor para un fluido en movimiento en lugar del uso de isotermas. Los tres casos de estudio se tomaron arbitrariamente por ser casos estudiados experimental y numéricamente, donde se conocen los campos de velocidad y temperatura, también por ser casos donde el régimen de trasferencia de calor es principalmente por convección. Se encontró que las isotermas dejan de ser una herramienta de visualización efectiva para los casos donde el fluido se encuentra en movimiento, mientras que las líneas de calor lo caracterizan independientemente del valor del númerode Rayleigh.

Descriptores: visualización, transferencia de calor, isotermas, convección en cavidades, líneas de calor.

\begin{abstract}
The heatline visualization technique is presented for three cases of free convection in cavities. The advantages of the technique over the commonly used isotherms in fluid mechanics are shown and discussed. The three cases under study have been arbitrarily chosen because each one of them can be found experimentally and numerically in the open literature, providing results for flow and temperature fields. It was found that isotherms are not an effective visualization tool in fluid motion phenomena while heatlines characterize these flows regardless of the value of Rayleigh number.
\end{abstract}

Keywords: Visualization, heat transfer, isotherms, cavity convection, heatlines.

\section{Introducción}

Las líneas de calor constituyen una herramienta matemática para la visualización del vector flujo de calor en problemas de convección. Esta técnica fue propuesta inicialmente por Kimura y Bejan (1983). Estos autores señalaron que para problemas de conducción de calor en sólidos, las isotermas representan fielmente el campo de temperaturas en el sólido, mientras que para convección de calor donde el fenómeno se caracteriza por el movimiento de un fluido, las isotermas resultan más difíciles de interpretar y pueden conducir a conclusiones equivocadas, véase por ejemplo, Osorio et al. (2004).

Las líneas de calor son una clara analogía a las líneas de corriente que se utilizan con el fin de visualizar el flujo, además de reducir el número de variables y ecuaciones que describen la mecánica de fluidos. Es muy común graficar la función de corriente en función de las variables espaciales, mostrando así el recorrido de las partículas del flujo dentro del volumen de interés.

En estudios de transferencia de calor por convección se acostumbra presentar los resultados de velocidad y 
DOI: http://dx.doi.org/10.22201/fi.25940732e.2010.11n4.031

Cervantes de Gortari J.G., Torchia-Núñez J.C., Serrano-Gutiérrez J.A., Muñoz-Barrera A., Guzmán-Vázquez E., Gutiérrez-Coria F.,

Chávez-López O., Rosas-Yescas I. y Martínez-Galván E.

temperatura mediante funciones de corriente e isotermas, respectivamente. Los resultados numéricos para transferencia de calor por convección se presentan básicamente mediante distribuciones de variables como la velocidad y la temperatura, las cuales describen el estado del sistema localmente, pero no su estructura física asociada (Deng et al., 2005).

Sin embargo, una vez obtenida la velocidad y temperatura en cada punto, es posible construir las líneas de calor en el espacio. Las líneas de calor surgen como una necesidad de visualizar la trayectoria del flujo de calor en un campo de flujo que involucre transferencia de calor por convección y conducción. Algunos estudios en la línea de las líneas de calor pueden encontrarse en la literatura especializada como Morega y Bejan (1993), Dalal y Kumar (2008), Mahmud y Fraser (2007), Basak et al. (2009), Basak et al. (2009b), Basak et al. (2009c), Mobedi y Oztop (2008) y Zhao et al. (2008).

\section{Desarrollo}

\section{Propiedades de la función $\mathrm{H}$}

Las líneas de calor son los lugares geométricos formados por aquellos puntos donde el vector flujo de calor resulta tangente a la línea en todo punto. El flujo de calor está compuesto por dos términos: el primer término es el advectivo $\rho c_{p} V T$. Este término es el transporte de entalpía que se mueve con el fluido. El segundo término $k T$, es el término de difusión térmica.

Debido al término advectivo (de movimiento) las líneas de calor no son, en general, perpendiculares a las isotermas. Solamente lo serían en el caso de que la velocidad del fluido fuera igual a cero; es decir, que se tratara de un régimen puramente conductivo de transferencia de calor. Las isotermas son líneas donde cada punto posee la misma temperatura; son muy utilizadas en meteorología y termodinámica de la atmósfera. Asimismo, son de gran utilidad en el área de la conducción de calor, ya que describen la distribución de temperaturas en el sólido.

Sin embargo, cuando se trata de un fluido en movimiento, las isotermas dejan de ser perpendiculares al vector flujo de calor $q$ y aunque siguen representando líneas de temperatura constante, es el flujo de calor lo que se quiere visualizar. Por la misma razón, en mecánica de fluidos se utilizan las líneas de corriente en vez de las líneas isobáricas (presión constante) porque es de mayor interés visualizar el flujo que los potenciales (gradientes de propiedades intensivas) que lo producen.

Se comenzará por definir la función de calor para una configuración bidimensional en coordenadas cartesianas.
Los vectores de flujo de calor en convección pueden expresarse según:

$$
\begin{aligned}
& q_{x}=\rho c_{p} u T-k \frac{\partial T}{\partial x} \\
& q_{y}=\rho c_{p} v T-k \frac{\partial T}{\partial y}
\end{aligned}
$$

donde $q y$ es el flujo de calor en la dirección $y$ y $q x$ es el flujo de calor en la dirección $x$. Las propiedades del fluido son: la conductividad térmica del fluido $k$ en $\mathrm{W} / \mathrm{mK}$, $c_{p}$ es la capacidad calorífica específica a presión constante en $\mathrm{kJ} / \mathrm{kgK}$, $\rho$ es la densidad en $\mathrm{kg} / \mathrm{m}^{3}$, y $T$ es la temperatura en $\mathrm{K}$. Las componentes $u$ y $v$ del vector velocidad en la dirección $x$ e $y$, respectivamente, en $\mathrm{m} / \mathrm{s}$.

Ahora introducimos la función $H$ que se define como:

$$
\begin{aligned}
& q_{x}=\frac{\partial H}{\partial y} \\
& q_{y}=\frac{\partial H}{\partial x}
\end{aligned}
$$

La función $H$ satisface la ecuación de conservación de la energía para la convección en estado permanente de un fluido con propiedades constantes:

$$
u \frac{\partial T}{\partial x}+v \frac{\partial T}{\partial y}=\alpha\left(\frac{\partial^{2} T}{\partial x^{2}}+\frac{\partial^{2} T}{\partial y^{2}}\right)
$$

A continuación, separamos los términos con respecto a las derivadas

$$
\frac{\partial}{\partial x}\left(u T-\alpha \frac{\partial T}{\partial x}\right)+\frac{\partial}{\partial y}\left(v T-\alpha \frac{\partial T}{\partial y}\right)=0
$$

Se puede observar que los términos entre paréntesis de la ecuación (6) corresponden a las expresiones (3) y (4). Con esto, se asegura que la función definida de esta manera satisfaga la ecuación de la energía (5).

La ecuación de una línea de calor se encuentra cuando se hace $H$ igual a una constante. Al ser $H$ una función de $x$ e $y$, en general, el cambio total de esta función se expresa como:

$$
d H=\frac{\partial H}{\partial x} d x+\frac{\partial H}{\partial y} d y
$$

Sustituyendo (3) y (4) en (7) resulta 
DOI: http://dx.doi.org/10.22201/fi.25940732e.2010.11n4.031

Líneas de calor para convección natural en cavidades cerradas: Tres casos de estudio

$d H=-q_{y} d x+q_{x} d x$

Entonces de la ecuación (8), la ecuación de la línea de calor $H=$ constante será

$0=-q_{y} d x+q_{x} d y$

Despejando, se obtiene:

$$
\left(\frac{d y}{d x}\right)_{H}=\frac{q_{y}}{q_{x}}
$$

La ecuación (10) es la definición de línea de calor. De aquí que un valor distinto de la constante $H$ define una línea de calor diferente. Nótese que las líneas de calor se definen para un flujo en régimen permanente, debido a que el tiempo no interviene. Al igual que las líneas de corriente, en el caso en que existiera flujo transitorio, las líneas de calor se representan para un instante de tiempo particular.

\section{Resultados}

\section{Casos de estudio}

En este estudio se empleó el lenguaje de programación PHOENICS para la obtención de las soluciones numéricas de las ecuaciones acopladas de cantidad de movimiento y energía para cada uno de los casos a tratar. Este paquete comercial se fundamenta en la discretización de las variables mediante volumen finito para la solución de las ecuaciones de conservación de energía y masa en cada punto de la región de estudio. También se utiliza este paquete para resolver las ecuaciones de las líneas de corriente, de calor y conducción, proporcionando además de los campos de velocidad, temperatura, así como las líneas de corriente, calor y conducción en cada uno de los casos de estudio.

El tamaño de la malla es de $200 \times 200$ para todos los casos. El tamaño de la malla se justifica por el tiempo de procesamiento y precisión de los resultados. La solución numérica para cada uno de los casos tomaba aproximadamente una hora de tiempo de procesamiento. Se validó el algoritmo utilizando un caso sencillo de convección natural en cavidades cerradas que aparece en Kimura y Bejan (1983). Se utilizó agua como fluido de trabajo para cada uno de los casos. La temperatura máxima y mínima son $302.84 \mathrm{~K}$ y $293.15 \mathrm{~K}$, respectivamente. Las dimensiones de las cavidades son de $0.01 \times 0.01 \mathrm{~m}$. Con estos datos el número de Rayleigh es igual a $1.98 \mathrm{x}$
$10^{4}$ (Bejan, 2004). El número de Rayleigh es un parámetro adimensional que relaciona los efectos de flotación con los efectos viscosos en un sistema donde se presenta convección natural y se calcula en este trabajo según la siguiente expresión matemática:

$$
R a=\frac{g \beta \Delta T L^{3}}{v \alpha}
$$

A medida que el valor del número de Rayleigh se incrementa, la fuerza de flotación que se produce en un fluido, debido a la diferencia de densidades, supera a la resistencia que ejerce la sustancia al movimiento, debido a la viscosidad. La diferencia de densidades en el seno del fluido se debe, en este trabajo, a la diferencia de temperaturas entre las paredes de la cavidad. Una vez establecido el fluido y la diferencia de temperaturas, el número de Rayleigh varía directamente con la velocidad del flujo, por lo que tiene influencia directa con el tipo de régimen en el que se encuentra el flujo. Para este caso en particular, el valor del número de Rayleigh indica que se está en régimen laminar para todos los casos. En general, el valor del número de Rayleigh para el cual el flujo se vuelve turbulento depende de la configuración geométrica, i.e. $\mathrm{Ra}=10^{9} \mathrm{y} \mathrm{Ra}=47,000$ para placas verticales y horizontales, respectivamente (Eckert y Drake, 1972).

\section{Cavidad con particiones}

Los bafles o particiones tienen una altura de $0.007 \mathrm{~m}$ colocados a 0.0025 y $0.0075 \mathrm{~m}$ respecto a la pared lateral; el bafle izquierdo está unido a la pared superior y el otro a la pared inferior. La pared lateral izquierda está a mayor temperatura que la pared lateral derecha. Los bafles se modelan como superficies adiabáticas, al igual que la pared inferior y superior. En la figura 1 se puede visualizar tanto el flujo de materia dentro de la cavidad como el transporte de calor. Mediante las líneas de corriente (figura $1^{a}$ ), se observa que el adelgazamiento entre líneas de corriente implica una aceleración del fluido y la formación de una celda convectiva que comienza en la parte inferior de la pared lateral izquierda hasta la parte superior de la pared lateral derecha con una dirección de giro antihorario. Estas observaciones están de acuerdo con los trabajos de algunos autores recopilados por Bejan (2004) como Nansteel et al. (1981) y Nienchuan et al. (1983).

Las isotermas en la figura $1 \mathrm{~b}$ muestran que tanto el fluido entre las paredes laterales y los bafles se encuentra casi en equilibrio térmico, por lo que podríamos 
DOI: http://dx.doi.org/10.22201/fi.25940732e.2010.11n4.031

Cervantes de Gortari J.G., Torchia-Núñez J.C., Serrano-Gutiérrez J.A., Muñoz-Barrera A., Guzmán-Vázquez E., Gutiérrez-Coria F.,

Chávez-López O., Rosas-Yescas I. y Martínez-Galván E.

inferir que no existe movimiento en esas regiones. Sin embargo, como ya se dijo, las isotermas no resultan herramientas de visualización suficientemente claras en problemas donde existe movimiento de un fluido. Se debe recurrir a la deducción de las isotermas más que a la simple observación de líneas que representan trayectorias en el desplazamiento de materia o energía.

Las líneas de calor mostradas en la figura $1 \mathrm{~d}$ son, en este caso, muy parecidas a las líneas de corriente, ya que el número de Rayleigh es alto. Estas líneas de calor muestran por dónde y con qué intensidad viaja el calor al provocarse un gradiente de temperaturas entre paredes laterales. Nótese cómo las líneas de calor salen y llegan perpendiculares a las paredes laterales, debido a que en las paredes se tiene únicamente el proceso de difusión.

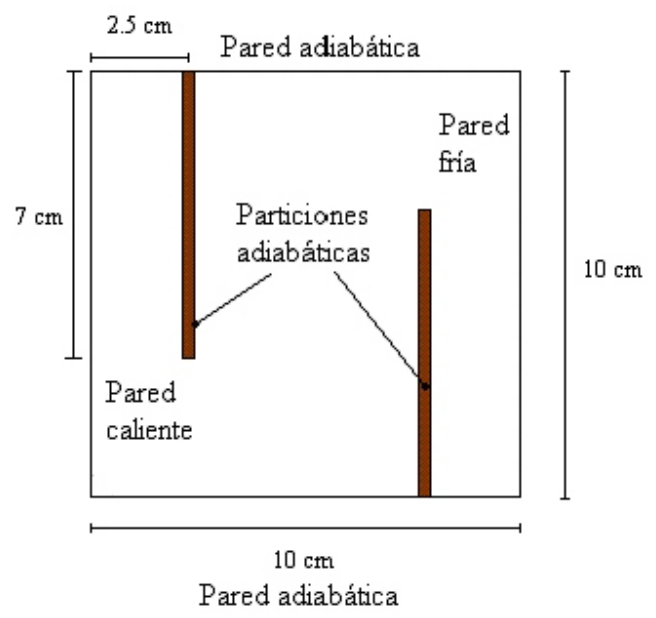

Figura 1a. Esquema de la cavidad

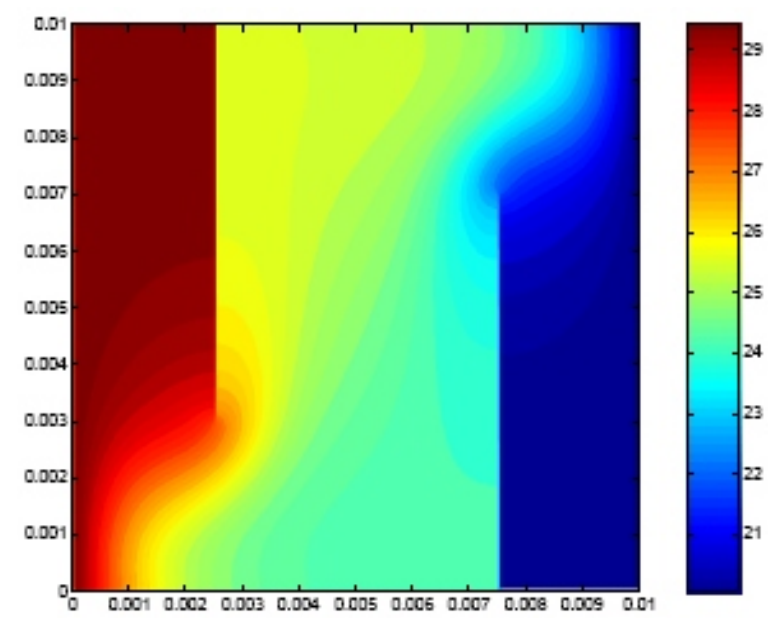

Figura 1c. Isotermas
Asimismo, las líneas de calor no tocan las superficies adiabáticas como son los bafles y las paredes superior e inferior. Esto ocurre porque no existe transferencia de calor en dirección perpendicular a las paredes (condición adiabática) y por lo tanto, las isotermas son perpendiculares a la pared (figura 1d).

\section{Cavidad con calentamiento discreto en el fondo y paredes laterales enfriadas}

La fuente discreta de calor localizada en el centro de la pared inferior está a mayor temperatura que las paredes laterales frías. El resto de la pared inferior se considera adiabática como la pared superior.

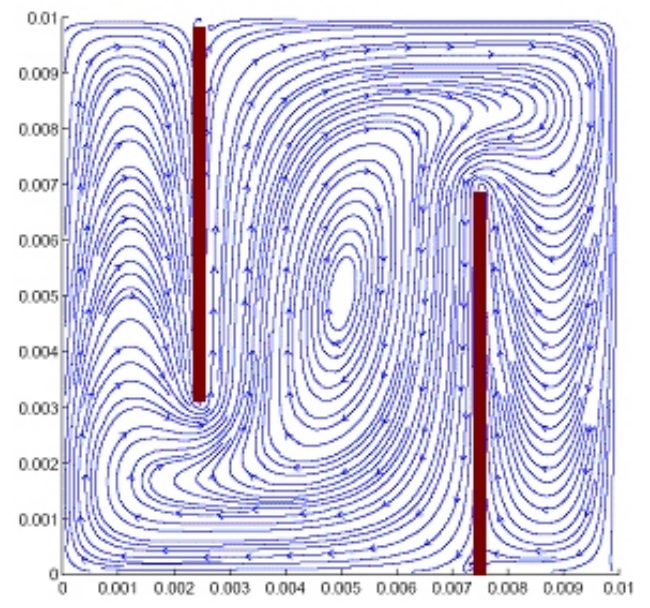

Figura 1b. Lineas de corriente

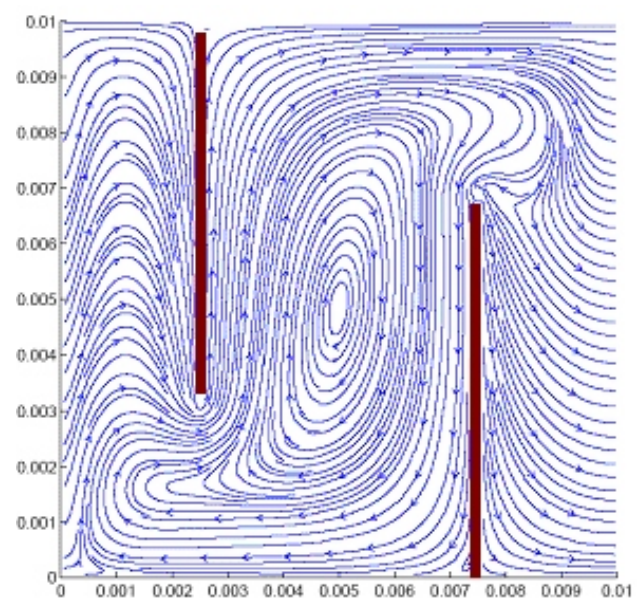

Figura 1d. Lineas de calor 
La fuente discreta de calor produce un calentamiento en el fluido que está en contacto con ella y con esto una fuerza de flotación que produce un movimiento vertical en contra de la gravedad en el centro de la cavidad. La estructura del flujo para este valor del número de Rayleigh es un par de celdas simétricas donde el fluido caliente sube por el centro de la cavidad y el fluido frío baja cerca de las paredes laterales.

Este caso de estudio fue elaborado experimental y numéricamente por Calcagni et al. (2005). Se agregaron las líneas de calor para mostrar su utilidad como herramienta de visualización. Como puede verse en la figura $2 \mathrm{~d}$ el calor es transportado casi enteramente por convección; esto es evidente comparando las figuras $2 \mathrm{a}$ y 2d; las líneas de calor son muy similares a las líneas de

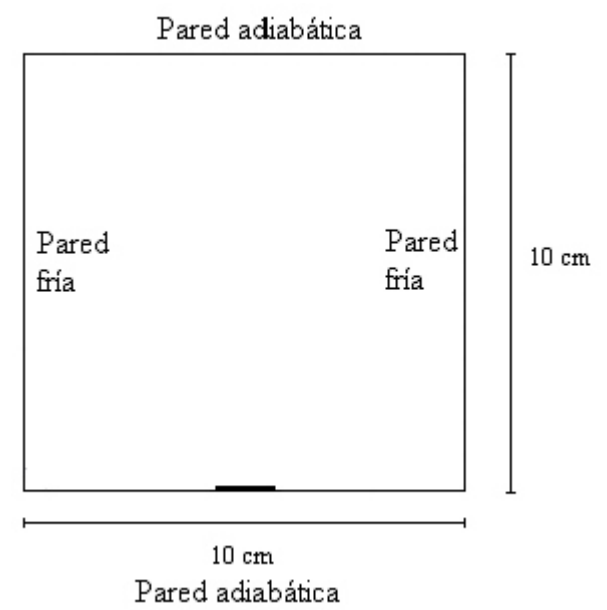

Figura 2a. Esquema de la cavidad

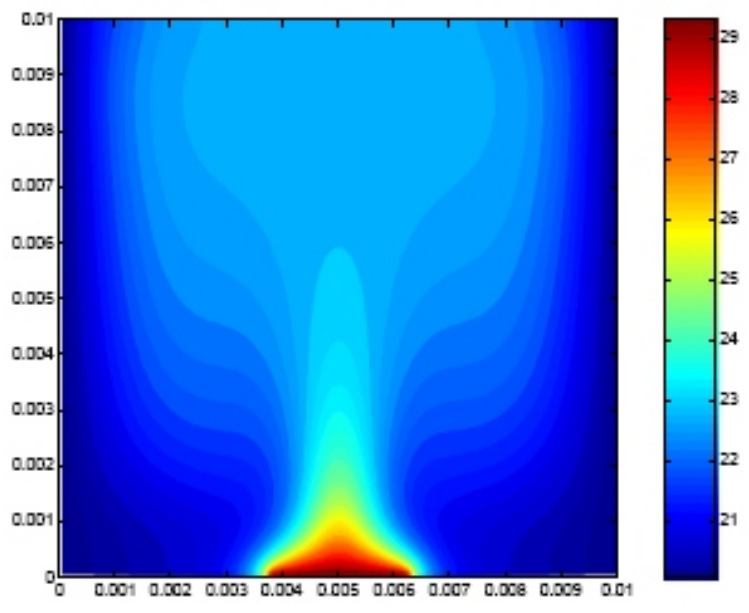

Figura 2c. Isotermas corriente, excepto cerca de las paredes laterales donde la conducción de calor es dominante. El calor se transporta desde la fuente de calor por convección desde el centro de la cavidad hacia arriba, y una vez que pierde energía o llega a la pared superior adiabática se dirige hacia el sumidero que, en este caso, son las paredes laterales frías. Es evidente una zona cercana a las paredes laterales donde el transporte de calor es predominantemente por difusión.

\section{Cavidad con un cuerpo a mayor temperatura $y$ paredes laterales a menor temperatura}

La barra circular tiene $0.0025 \mathrm{~m}$ de diámetro y está colocada en el centro de la cavidad. La barra está a mayor

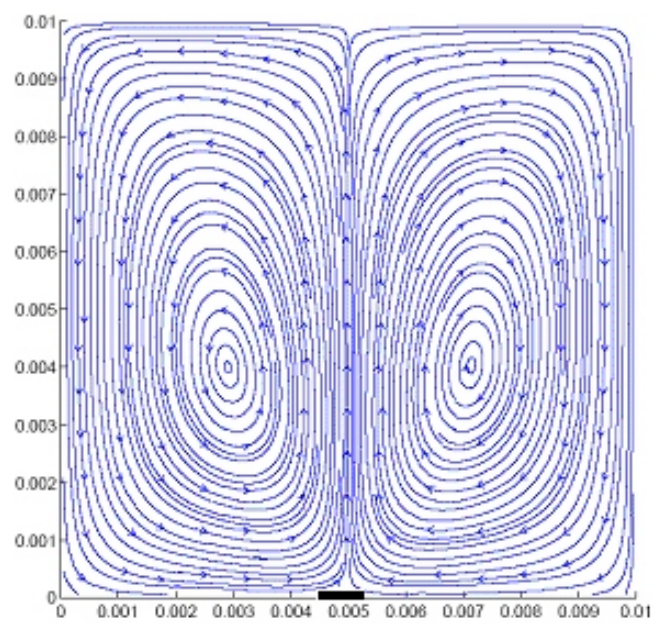

Figura 2b. Lineas de corriente

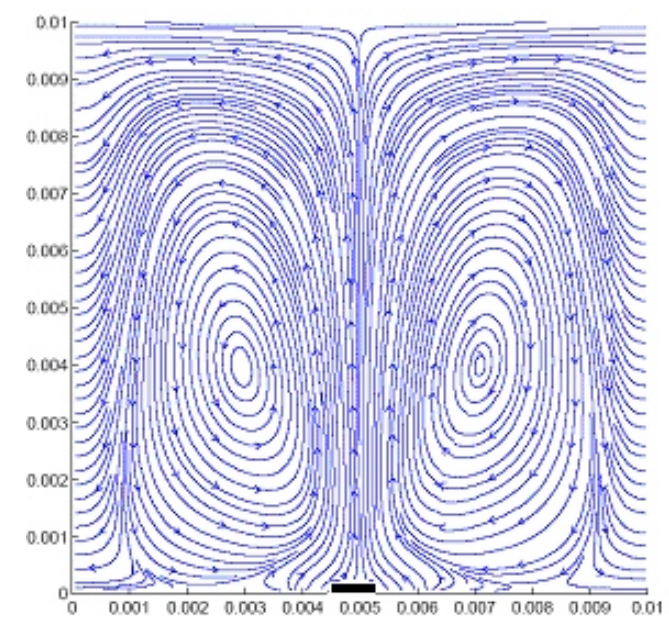

Figura 2d. Lineas de calor 
DOI: http://dx.doi.org/10.22201/fi.25940732e.2010.11n4.031

Cervantes de Gortari J.G., Torchia-Núñez J.C., Serrano-Gutiérrez J.A., Muñoz-Barrera A., Guzmán-Vázquez E., Gutiérrez-Coria F., Chávez-López O., Rosas-Yescas I. y Martínez-Galván E.

temperatura que las paredes laterales frías. Las paredes inferior y superior son adiabáticas. En este caso, el cuerpo a mayor temperatura es el cilindro en el centro de la cavidad (representado por una circunferencia suponiendo bidimensionalidad) y las paredes laterales son el sumidero de calor. Las paredes superior e inferior son adiabáticas. Mediante las líneas de corriente distinguimos dos celdas convectivas encima de la línea de centro del cilindro.

El espaciamiento entre líneas de corriente muestran que cerca del centro de las celdas la velocidad del fluido es mayor, que lejos del centro o que en la parte inferior de la celda.

Las isotermas claramente muestran la región de mayor temperatura y actividad; si se toma en cuenta que el

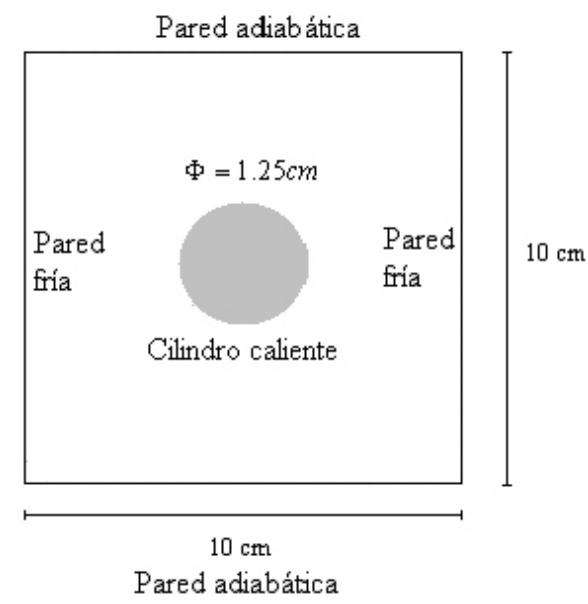

Figura 3 a. Esquema de la cavidad

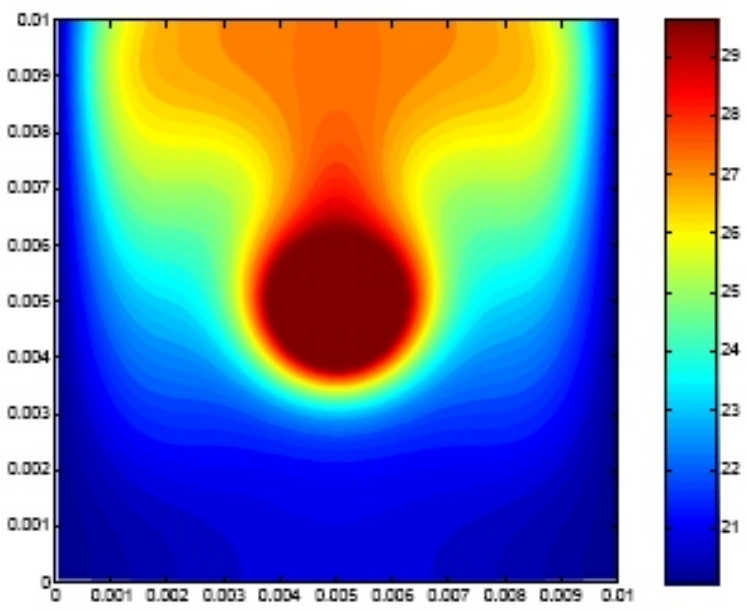

Figura 3c. Isotermas flujo de calor es perpendicular a las isotermas, las líneas de calor serían radiales a la esfera.

Cuando una cantidad de fluido caliente (pluma térmica) llega a la pared adiabática, la región de alta temperatura se ensancha encima del cilindro. Las líneas de calor muestran una pequeña región alrededor del cilindro, donde la conducción domina el transporte de calor, lejos de éste se forman celdas donde se transporta el calor con mayor intensidad como ocurre encima del cilindro.

Para este último caso de estudio se agregan dos figuras (3e y 3f), con el fin de realizar una comparación cualitativa de las líneas de calor con otras líneas utilizadas en el estudio de la mecánica de fluidos (líneas de corriente) y conducción de calor (líneas de flujo de calor).

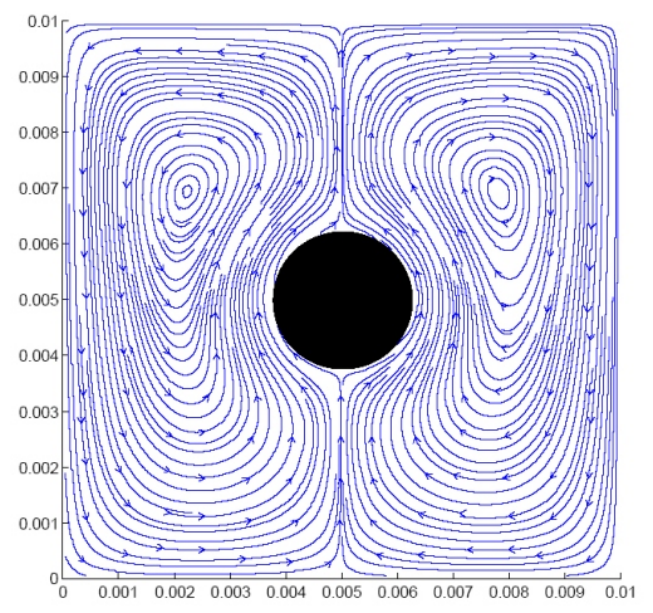

Figura 36. Lineas de corriente

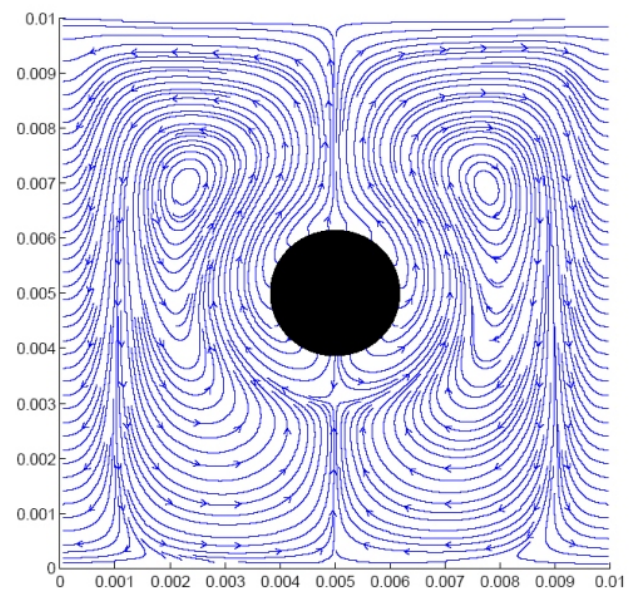

Figura $3 d$. Lineas de calor 
DOI: http://dx.doi.org/10.22201/fi.25940732e.2010.11n4.031

Líneas de calor para convección natural en cavidades cerradas: Tres casos de estudio

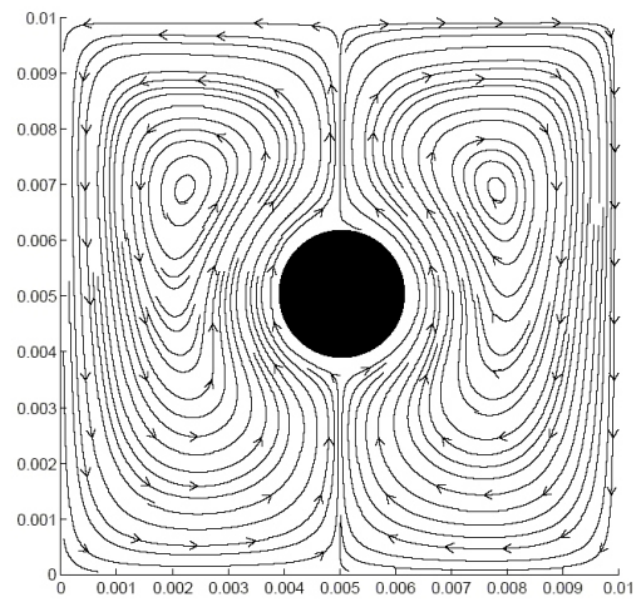

Figura 3e. Lineas de corriente

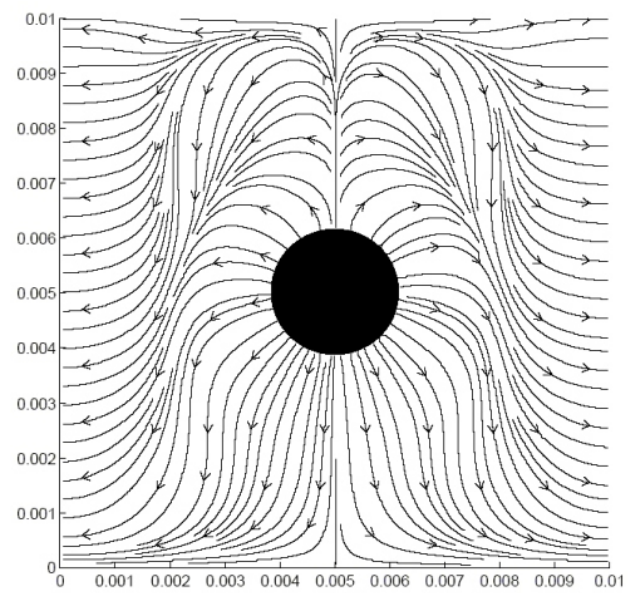

Figura 3e. Lineas de flujo de calor para difusión pura
Se pueden comparar las figuras $3 \mathrm{~d}$ y $3 e$, donde se observa que para este régimen en particular las líneas de corriente son paralelas, casi idénticas a las líneas de calor cuando el fluido está lejos de las superficies, lo cual indica que se trata de un fenómeno dominado por advección. Sin embargo, es notable la diferencia que existe entre ambas líneas cerca de las superficies. Por otra parte, la figura $3 \mathrm{f}$ muestra las líneas de conducción (o líneas de flujo de calor). Como vimos, la función de calor está compuesta por el flujo de entalpía y el flujo de calor mediante conducción. Esto implica que serán iguales cuando la velocidad sea igual a cero o la conducción de calor domine sobre la advección. Esta situación ocurre cerca de las paredes laterales que actúan como sumideros de calor. En cambio, en el resto de la cavidad las líneas de conducción son casi perpendiculares a las líneas de calor.

\section{Conclusiones}

Como ya se ha mencionado, las líneas de calor son una forma de visualizar el flujo de calor en un fenómeno regido, pero no exclusivamente por la convección.

Así como se define la función de corriente para visualizar la ruta que siguen las partículas de un fluido en movimiento, las líneas de calor muestran la ruta en la que fluye la energía. Es común que, de forma intuitiva pero errónea, se piense que la transferencia de calor en un fenómeno de convección podría ser análoga a la transferencia de calor por conducción, donde el flujo de calor es perpendicular a las isotermas.

Las líneas de calor tienen dos componentes que representan dos formas de transportar calor: conducción y advección. En la primera, las partículas del fluido transmiten calor a las demás partículas adyacentes, con las cuales están en contacto. Esto ocurre en las partículas del fluido que están en contacto con una pared, donde las líneas de calor son perpendiculares a la misma, ya que debido a la condición de no deslizamiento la transferencia de calor es únicamente por conducción.

En el fenómeno de advección, dado que el fluido está en movimiento, éste transporta un flujo de energía, la energía asociada a cada partícula. Por lo tanto, las líneas de calor o la ruta que siga el flujo de calor depende de dos cosas: la velocidad del fluido y la conductividad del mismo.

La forma que tomen los patrones de las líneas de calor dependerá de cuál sea la parte dominante en el fenómeno. Si el fluido tiene una conductividad térmica muy alta, el calor se transmitirá de forma rápida de una partícula de fluido a otra, por lo que las líneas de calor tenderán a ser más perpendiculares a las isotermas, es decir, en el límite el flujo de calor será prácticamente por pura conducción, ya que antes que la partícula de fluido cambie de posición se habrá dado una transferencia de calor entre partículas, minimizando el efecto de la velocidad y acercando el fenómeno a lo que sucede en un sólido.

Suponiendo que la velocidad del fluido sea relativamente alta y su conductividad térmica baja, la transferencia de calor se deberá prácticamente al movimiento de las partículas de fluido; es decir, podría decirse que casi no hay transferencia de calor entre partículas del mismo fluido y el transporte de calor se realice únicamente por el movimiento del fluido, por lo que las líneas de calor tenderían a seguir a las líneas de corriente, excepto en aquellas zonas cercanas a cuerpos sólidos o 
DOI: http://dx.doi.org/10.22201/fi.25940732e.2010.11n4.031

Cervantes de Gortari J.G., Torchia-Núñez J.C., Serrano-Gutiérrez J.A., Muñoz-Barrera A., Guzmán-Vázquez E., Gutiérrez-Coria F.,

Chávez-López O., Rosas-Yescas I. y Martínez-Galván E.

de estancamiento, donde la conducción se debe tomar en cuenta porque la velocidad del flujo se reduce a cero.

En este trabajo, las líneas de calor muestran la trayectoria que siguen cuando se transportan en cavidades. Así, existirán regiones donde predominen, ya sea un régimen conductivo o un régimen convectivo, dependiendo de las condiciones del fenómeno y de las propiedades del fluido. Las líneas de calor son una buena herramienta, tanto para regímenes de transferencia de calor por conducción como para la advección, dando una clara imagen de cómo fluye el calor.

\section{Agradecimientos}

Esta investigación fue patrocinada parcialmente por PAPIIT-UNAM, a través del proyecto IN115703-2, así como por la DGAPA-UNAM con los proyectos IN103106, IN101808 y PE101007.

\section{Referencias}

Basak T., Aravind G. y Roy S. Visualization of Heat Flow Due to Natural Convection within Triangular Cavities Using Bejan's Heatline Concept. Int. J. Heat Mass Trans., 52 (11-12):2824-2833. 2009b.

Basak T., Roy S. y Pop I. Heat Flow Analysis for Natural Convection within Trapezoidal Enclosures Based on Heatline Concept. Int. J. Heat Mass Trans., 52(11-12): 2471-2483. 2009.

Basak T., Roy S. y Aravind G. Analysis of Heat Recovery and Thermal Transport within Entrapped Fluid Based on Heatline Approach. Chem. Eng. Sci., 64(8):1673-1686. 2009c.

Bejan A. Convective Heat Transfer. Third Ed. New York. John Wiley \& Sons. 2004.
Calcagni B., Marsili F. y Paroncini M. Natural Convective Heat Transfer in Square Enclosures Heated from Below. Applied Thermal Engineering, 25:2522-2531. 2005.

Dalal A. y Kumar M. Heatline Method for the Visualization of Natural Convection in a Complicated Cavity. Int. J. Heat Mass Trans, 51(1-2):263-272. 2008.

Deng O-H y Tang G-F. Numerical Visualization of Mass and Heat Transport for Cconjugate Natural Convection/Heat Conduction by Streamline and Heatline. Int. J. Heat Mass Transf, 45(11):2373-2385. 2002.

Eckert E.R.G. y Drake R.M. Analysis of Heat and Mass Transfer. New York. McGraw-Hill. 1972.

Kimura S. y Bejan A. The Heatline Visualization of Convective Heat Transfer. J. Heat Transfer, 105:916-919. 1983.

Mahmud S. y Fraser R.A. Visualizing Energy Flows Through Energy Streamlines and Pathlines. Int. J. Heat Mass Trans, (19-20):3990-4002. 2007.

Mobedi M. y Oztop H.F. Visualization of Heat Transport Using Dimensionless Heatfunction for Natural Convection and Conduction in an Enclosure with Thick Solid Ceiling. Computers \& Mathematics with Applications, 56(10):2596-2608. 2008.

Morega A.I. y M., Bejan A. Heatline Visualization of Forced Convection Boundary Layers. Int. J. Heat Mass Trans, 36:3957-3966. 1993.

Nansteel M.W. y Greif R. Natural Convection in Undivided and Partially Divided Rectangular Enclosures. J. Heat Transfer, 103:623-629. 1981.

Nienchuan N.L. y Bejan A. Natural Convection in a Partially Divided Enclosure. Int. J. Heat Mass Trans, 26:1867-1878. 1983.

Osorio A., Ávila R. y Cervantes J.G. On the Natural Convection of Water Near its Density Inversion in an Inclined Square Cavity. Int. J. Heat Mass Trans, 47:4491-4495. 2004.

Zhao F., Liu D. y Tang G. Natural Convection in an Enclosure with Localized Heating and Salting from Below. Int. J. Heat Mass Trans., 51(11-12):2889-2904. 2008. 
DOI: http://dx.doi.org/10.22201/fi.25940732e.2010.11n4.031

Líneas de calor para convección natural en cavidades cerradas: Tres casos de estudio

\section{Semblanza de los autores}

Jaime G. Cervantes de Gortari. Se graduó como ingeniero mecánico electricista por la Universidad Nacional Autónoma de México (UNAM) en 1970. Obtuvo su maestría en ingeniería mecánica por la misma institución en 1972 y su doctorado (Ph.D) en ingeniería mecánica por la Purdue University, EUA en 1976. Es profesor titular "C" de tiempo completo, definitivo de la Facultad de Ingeniería de la UNAM.

Juan Cristóbal Torchia-Núñez. Es maestro en ingeniería egresado de la Facultad de Ingeniería de la UNAM. Actualmente realiza estudios de doctorado en termofluidos en la Facultad de Ingeniería, bajo la dirección del Dr. Cervantes de Gortari.

Juan Ángel Serrano-Gutiérrez. Es maestro en ingeniería egresado de la Facultad de Ingeniería de la UNAM. Desarrolla su investigación en métodos numéricos aplicados a la mecánica de fluidos.

Abraham Muñoz-Barrera. Es maestro en ingeniería egresado de la Facultad de Ingeniería de la UNAM. Actualmente trabaja en la industria automotriz.

Enrique Guzmán-Vázquez. Es licenciado en física por la Universidad Iberoamericana y doctor en ingeniería por la Facultad de Ingeniería de la UNAM.

Federico Gutiérrez-Coria. Es maestro en ingeniería egresado de la Facultad de Ingeniería de la UNAM. Es profesor de Laboratorio de Termofluidos en la Facultad de Ingeniería y actualmente realiza el doctorado en ingeniería mecánica.

Óscar Chávez-López. Es maestro en ingeniería egresado de la Facultad de Ingeniería de la UNAM. Actualmente realiza el doctorado en ingeniería mecánica utilizando métodos numéricos en transferencia de calor.

Israel Rosas-Yescas. Es maestro en ingeniería egresado de la Facultad de Ingeniería de la UNAM. Actualmente realiza estudios de doctorado en el Instituto de Materiales en el área de Reología.

Eduardo Martínez-Gálvan. Es maestro en ingeniería egresado de la Facultad de Ingeniería de la UNAM. Se desempeñó como profesor de Laboratorio de Termolfuidos en la Facultad de Ingeniería de la UNAM. Actualmente realiza el doctorado en la Universidad de Navarra en Ingeniería Mecánica sobre desarrollo de métodos numéricos. 